\title{
BMJ Open Experiences of people taking opioid medication for chronic non-malignant pain: a qualitative evidence synthesis using meta-ethnography
}

\author{
Vivien P Nichols (D) , ${ }^{1,2}$ Francine Toye, ${ }^{3}$ Sam Eldabe, ${ }^{4}$ Harbinder Kaur Sandhu, ${ }^{1}$ \\ Martin Underwood, ${ }^{1}$ Kate Seers ${ }^{2}$
}

To cite: Nichols VP, Toye F, Eldabe S, et al. Experiences of people taking opioid medication for chronic nonmalignant pain: a qualitative evidence synthesis using meta-ethnography. BMJ Open 2020;10:e032988. doi:10.1136/ bmjopen-2019-032988

- Prepublication history and additional material for this paper are available online. To view these files, please visit the journal online (http://dx.doi. org/10.1136/bmjopen-2019032988).

Received 15 July 2019 Revised 13 December 2019 Accepted 07 January 2020

Check for updates

(C) Author(s) (or their employer(s)) 2020. Re-use permitted under CC BY. Published by BMJ.

${ }^{1}$ Warwick Clinical Trials Unit, Warwick Medical School, University of Warwick, Coventry, UK

${ }^{2}$ Warwick Research in Nursing, Division of Health Sciences, Warwick Medical School, University of Warwick, Coventry, UK

${ }^{3}$ Physiotherapy Research Unit, Nuffield Orthopaedic Centre,

Oxford University Hospitals NHS

Trust, Oxford, UK

${ }^{4}$ Department of Pain Medicine, The James Cook University Hospital, Middlesbrough, UK

Correspondence to

Mrs Vivien P Nichols;

V.P.Nichols@warwick.ac.uk

\section{ABSTRACT}

Objective To review qualitative studies on the experience of taking opioid medication for chronic non-malignant pain (CNMP) or coming off them.

Design This is a qualitative evidence synthesis using a seven-step approach from the methods of metaethnography.

Data sources and eligibility criteria We searched selected databases-Medline, Embase, AMED, Cumulative Index to Nursing and Allied Health Literature, PsycINFO Web of Science and Scopus (Science Citation Index and Social Science Citation Index)—for qualitative studies which provide patients' views of taking opioid medication for CNMP or of coming off them (June 2017, updated September 2018).

Data extraction and synthesis Papers were quality appraised using the Critical Appraisal Skills Programme tool, and the GRADE-CERQual (Grading of Recommendations Assessment, Development and Evaluation working group - Confidence in Evidence from Reviews of Qualitative research) guidelines were applied. We identified concepts and iteratively abstracted these concepts into a line of argument.

Results We screened 2994 unique citations and checked 153 full texts, and 31 met our review criteria. We identified five themes: (1) reluctant users with little choice; (2) understanding opioids: the good and the bad; (3) a therapeutic alliance: not always on the same page; (4) stigma: feeling scared and secretive but needing support; and (5) the challenge of tapering or withdrawal. A new overarching theme of 'constantly balancing' emerged from the data.

Conclusions People taking opioids were constantly balancing tensions, not always wanting to take opioids, and weighing the pros and cons of opioids but feeling they had no choice because of the pain. They frequently felt stigmatised, were not always 'on the same page' as their healthcare professional and felt changes in opioid use were often challenging.

Trial registration number 49470934; Pre-results.

\section{INTRODUCTION}

Chronic non-malignant pain (CNMP) affects between an estimated $11 \%$ and $20 \%$ of the population in Europe and USA and
Strengths and limitations of this study

- To our knowledge this is the first qualitative evidence synthesis of patients' experiences of taking opioid medications.

- Meta-ethnography provides a thorough, systematic way of synthesising qualitative findings across multiple studies.

- Meta-ethnography provides the reviewer's interpretation of second-order concepts.

- Using a GRADE-CERQual (Grading of Recommendations Assessment, Development and Evaluation working group - Confidence in Evidence from Reviews of Qualitative research) approach can assist in rating confidence in the review findings.

- Qualitative research that illuminates patients' perspectives can help to shape future approaches to opioid management.

can impact heavily on people's quality of life. ${ }^{12}$ Opioid medications are strong painkillers which have a well-established role in the treatment of acute and cancer pain; they have also been advocated for CNMP. Opioids can have distressing side effects as dosages increase, such as constipation, sedation, drowsiness, nausea, decreased concentration and memory, or mood changes. ${ }^{3}$ Most people who use opioids develop tolerance to the painkilling effect of opioids, and some become dependent on them. Studies have shown that high opioid usage can also put people's lives at risk. ${ }^{4}$ Despite this, the prescription of opioid medication for CNMP has risen sharply in the higher income countries. Few studies of opioids have shown effectiveness beyond 12 weeks of follow-up. Population surveys have shown long-term use to be associated with increased side effects and limited pain relief. ${ }^{356}$

This synthesis of qualitative research was undertaken to underpin a process evaluation 
for the I-WOTCH (Improving the Wellbeing of people with Opioid Treated CHronic pain) study. I-WOTCH is a randomised controlled trial evaluating a multicomponent education and patient-centred group intervention with a one-to-one tapering programme against a control of an advice booklet with a relaxation CD. More information can be found in the main study protocol ${ }^{7}$ and process evaluation protocol. ${ }^{8}$ This qualitative evidence synthesis uses the methods of meta-ethnography to find out what people's experiences are of both using opioids for CNMP and their attempts to stop taking them.

\section{METHODS}

We use Noblit and Hare's ${ }^{9}$ seven stages of metaethnographic analysis. We used the new Meta- Ethnography Reporting Guidelines (eMERGe) to structure our report $^{10}$ (see online supplementary appendix 1 ). The protocol is published in the International Prospective Register of Systematic Reviews (PROSPERO) (http:// www.crd.york.ac.uk/PROSPERO).CRD42017082418

\section{Step 1: getting started}

In order to address what has been labelled an opioid epidemic, ${ }^{11}$ we need to understand people's experiences of being on opioids and of coming off them. Our team was chosen because of its expertise in primary qualitative research and qualitative evidence synthesis specific to chronic pain and opioid prescription.

\section{Step 2: deciding what is relevant}

We undertook systematic electronic searches in June 2017, with a rerun in September 2018, appraising relevant papers for quality using the Critical Appraisal Skills Programme (CASP) tool for qualitative research. ${ }^{12}$ One researcher (VPN) with the assistance of an academic librarian (SJ) searched seven electronic databases: Medline, Embase, Allied and Complementary Medicine Database (AMED), Cumulative Index to Nursing and Allied Health Literature (CINAHL), PsycINFO, Web of Science and Scopus (Science Citation Index and Social Science Citation Index). Forward citation searches were also conducted. We used search terms, free text and medical subject headings (MeSH) terms for all opioid drugs as well as their generic names. We combined these with the MeSH term 'pain' and a wide range of MeSH terms and words to describe all types of qualitative research and its analysis based on a search used by Toye et al in 2017. ${ }^{13}$ The search was limited to those in English and on humans, with no cut-off date. Online supplementary appendix 2 shows an example of our search terms.

Unique citations were screened independently by two researchers (VPN and ST; see the Acknowledgements section) against our inclusion and exclusion criteria (see box 1). Any disagreements were arbitrated by a third researcher (KS). Papers for full-text reading were identified and read by two researchers. Quality was assessed using the CASP tool. VPN critically appraised the studies

\section{Box 1 Inclusion and exclusion criteria}

Included studies.

- Adults (18 years or older) taking or have taken opioid medication in the last 5 years.

- Published in English in peer-reviewed journals with no time constraints.

- Must relate to patient perspectives on using opioid medication for chronic non-malignant pain.

- Must use qualitative methodology (any analytical approach) or mixed quantitative and qualitative methodology with qualitative findings reported separately.

- Where studies include participants with differing medication, we will include studies where the experience of those taking opioids is reported separately.

\section{Excluded studies.}

- Paediatric studies (age less than 18 years).

- Theoretical or methodological papers.

- Purely quantitative studies or mixed methods studies where the qualitative data are not presented separately.

- Studies concerning active cancer.

- Studies concerning headache.

- Studies concerning any acute, or acute postoperative, pain.

- Studies concerned only with healthcare professional or carer perspectives, or studies of mixed carer/patient/professional populations where patient perspectives are not presented separately.

- Non-English-language studies.

Theses or conference abstracts which are not peer-reviewed.

and KS independently appraised $10 \%$ for consistency. The CASP scores are shown in table 1 and online supplementary appendix 3. The GRADE-CERQual (Grading of Recommendations Assessment, Development and Evaluation working group - Confidence in Evidence from Reviews of Qualitative research) was used to appraise the reviewers' confidence in the research findings. ${ }^{145}$

\section{Step 3: reading the studies}

VPN read all the studies, and KS and FT read half of these papers each (so all were read twice), and all extracted the second-order concepts independently. A secondorder concept is a researcher's interpretation of data in a primary qualitative study. ${ }^{16} \mathrm{VPN}, \mathrm{KS}$ and FT met to discuss and reach agreement, and compiled a spreadsheet of all of the concepts extracted from the papers.

\section{Step 4: determining how the studies are related}

VPN sorted the concepts into categories by looking for any similarities and differences across all the studies. VPN, KS and FT discussed the categorisation of data on multiple occasions. To enable comparison across studies, VPN recorded descriptive data about each study (see table 1).

\section{Step 5: translating studies into each other}

Patterns and associations between categories were explored, and all researchers felt that a line of argument approach as defined by Noblit and Hare ${ }^{9}$ would be the most useful method to interpret the data. 


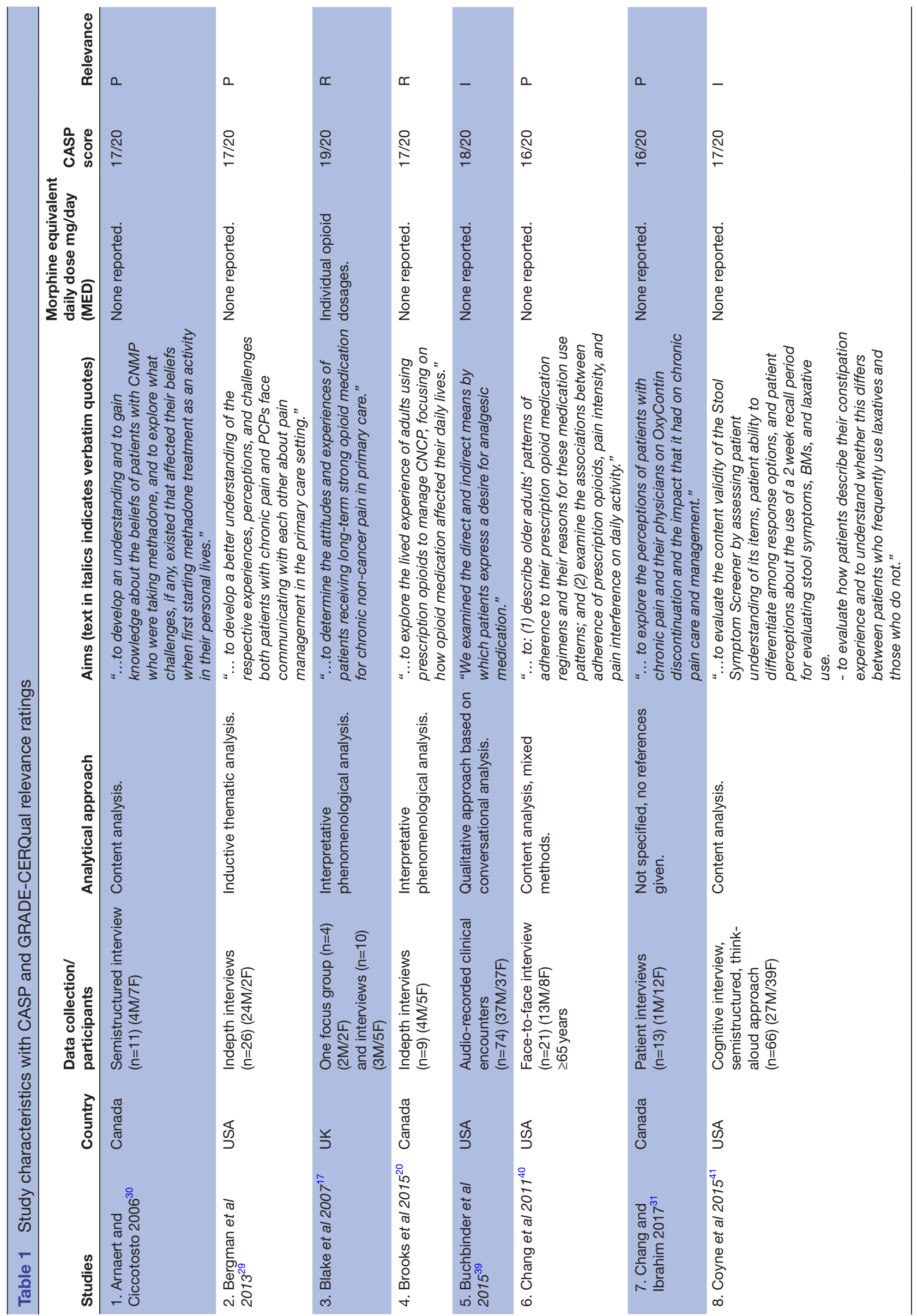




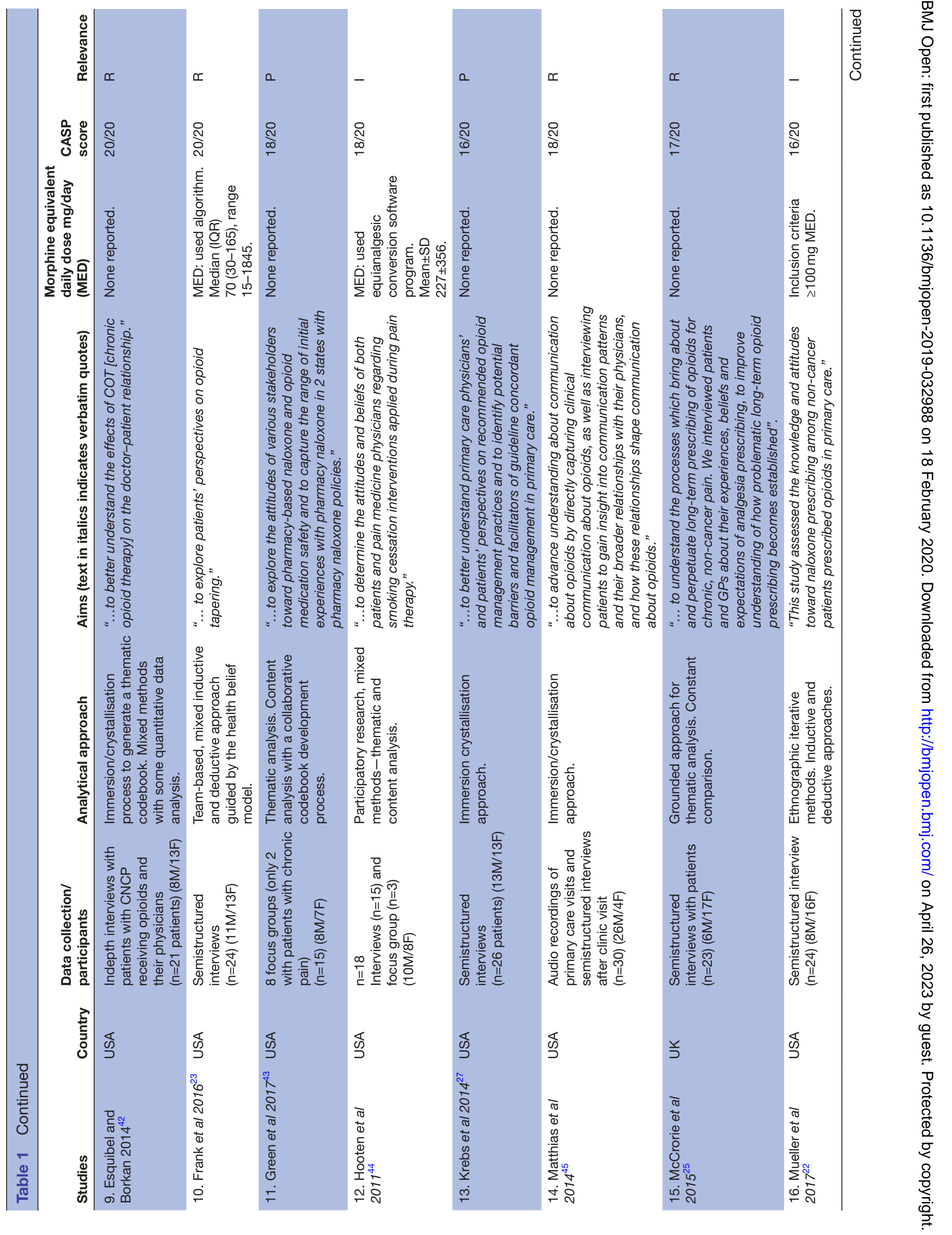




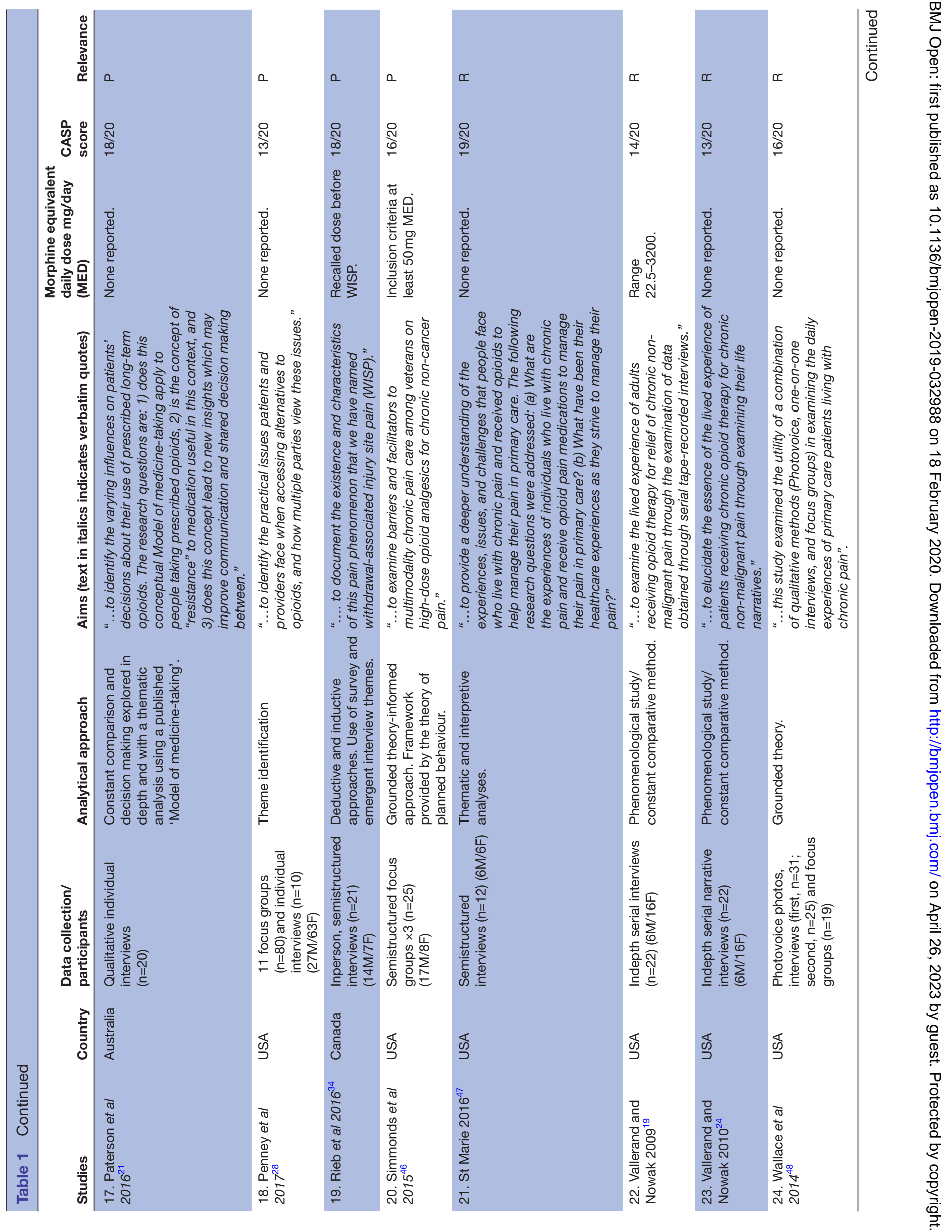




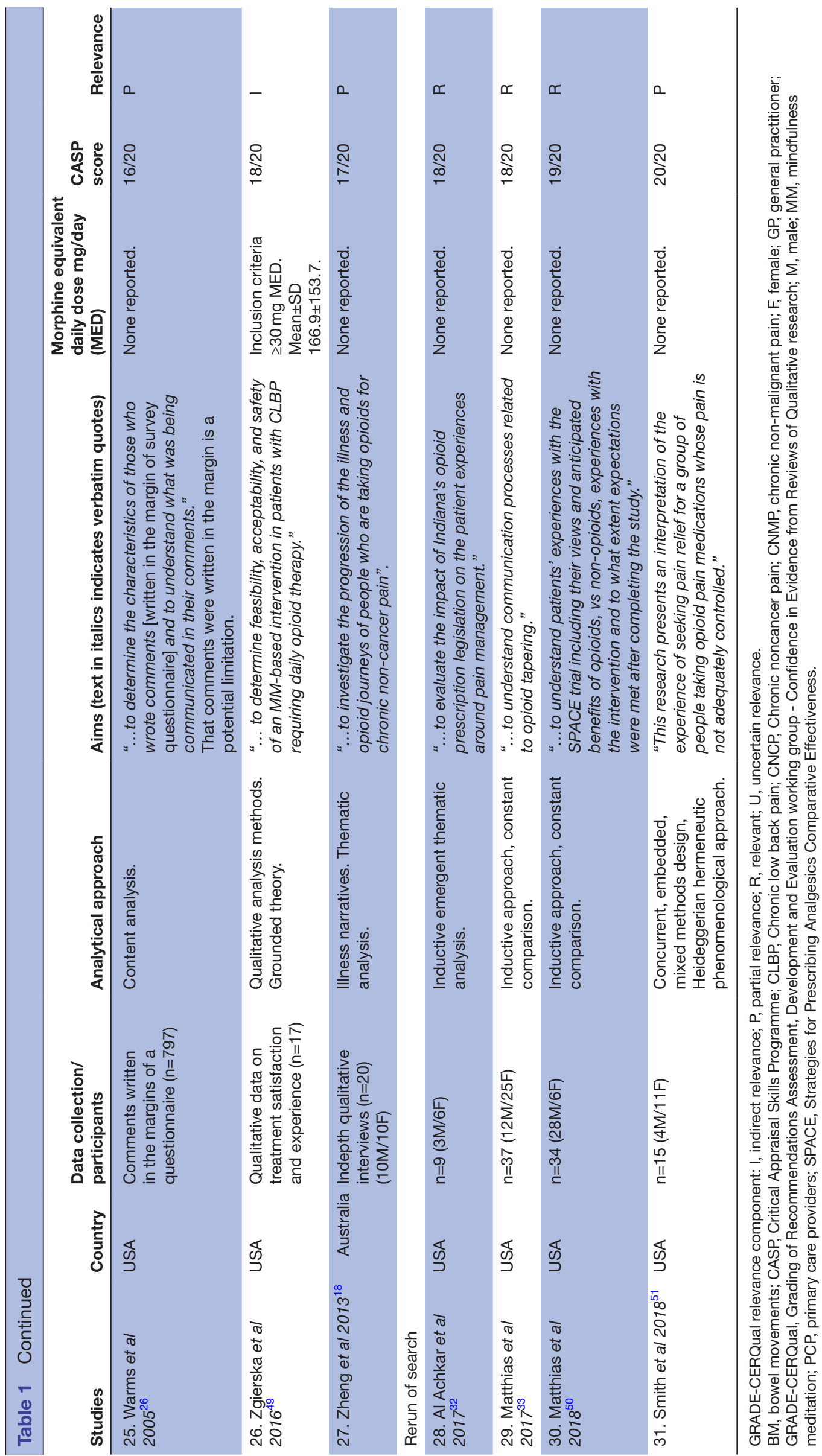

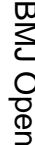

昙

음

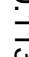




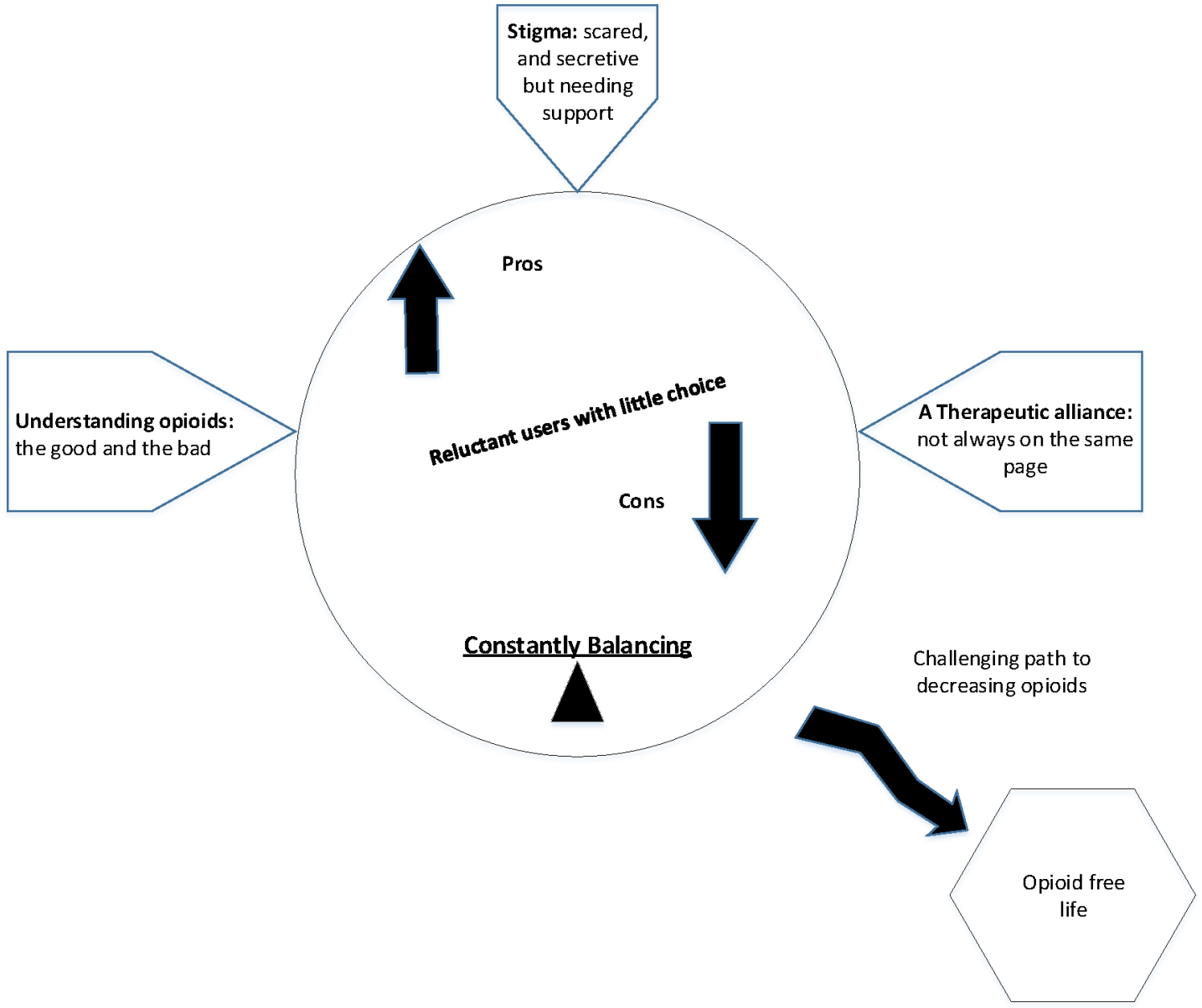

Figure 1 Concept model of the experiences of people taking opioid medication for chronic non-malignant pain.

\section{Step 6: synthesising translations}

Agreement was reached by clearly defining the overarching or third-order concepts arising from the data. A third-order concept is the reviewer's interpretation of second-order concepts.

\section{Step 7: expressing the synthesis}

We developed a conceptual model to show how the themes related to each other in a line of argument (see figure 1).

\section{Patient and public involvement}

We did not involve patients or the public in our work.

\section{RESULTS}

Two reviewers (VPN and ST) screened 2994 titles or abstracts (after the removal of duplicates from the 5064 citations retrieved) and identified 153 full texts of interest. Two reviewers (VPN and KS) read these and 122 were excluded. Reasons are given in the Preferred Reporting Items for Systematic Reviews and Meta-Analyses flow chart (see figure 2). The reviewers agreed to include 31 studies. The included studies were from the USA (23), Canada (4) UK (2) and Australia (2), and used a range of qualitative methods.
We report the four facets of GRADE-CERQual for all papers: (1) methodological, (2) confidence, (3) relevance and (4) adequacy of data (see tables 2 and 3).

\section{Synthesis of findings}

We abstracted five themes from the second-order concepts. Table 4 shows how each study contributed to each theme. We have illustrated each concept with exemplary quotations.

\section{Reluctant users with little choice}

This describes a resistance or hesitancy to take opioids mainly due to concerns about side effects or addiction, although they felt there were no other options available.

I don't want to become addicted, if I'm going to become addicted then as far as I'm concerned I'm a druggie, so I might as well not be here anyway, so I don't want to become addicted.... (Blake et al, p103) ${ }^{17}$

I just didn't want to go on them because I mean once you get on them that's it, you're sort of stuck on them. I didn't want to take morphine at first because there was a girl that I went through one of the courses with and she always seemed really dopey and drugged up so it took them a long while to talk me to into taking 

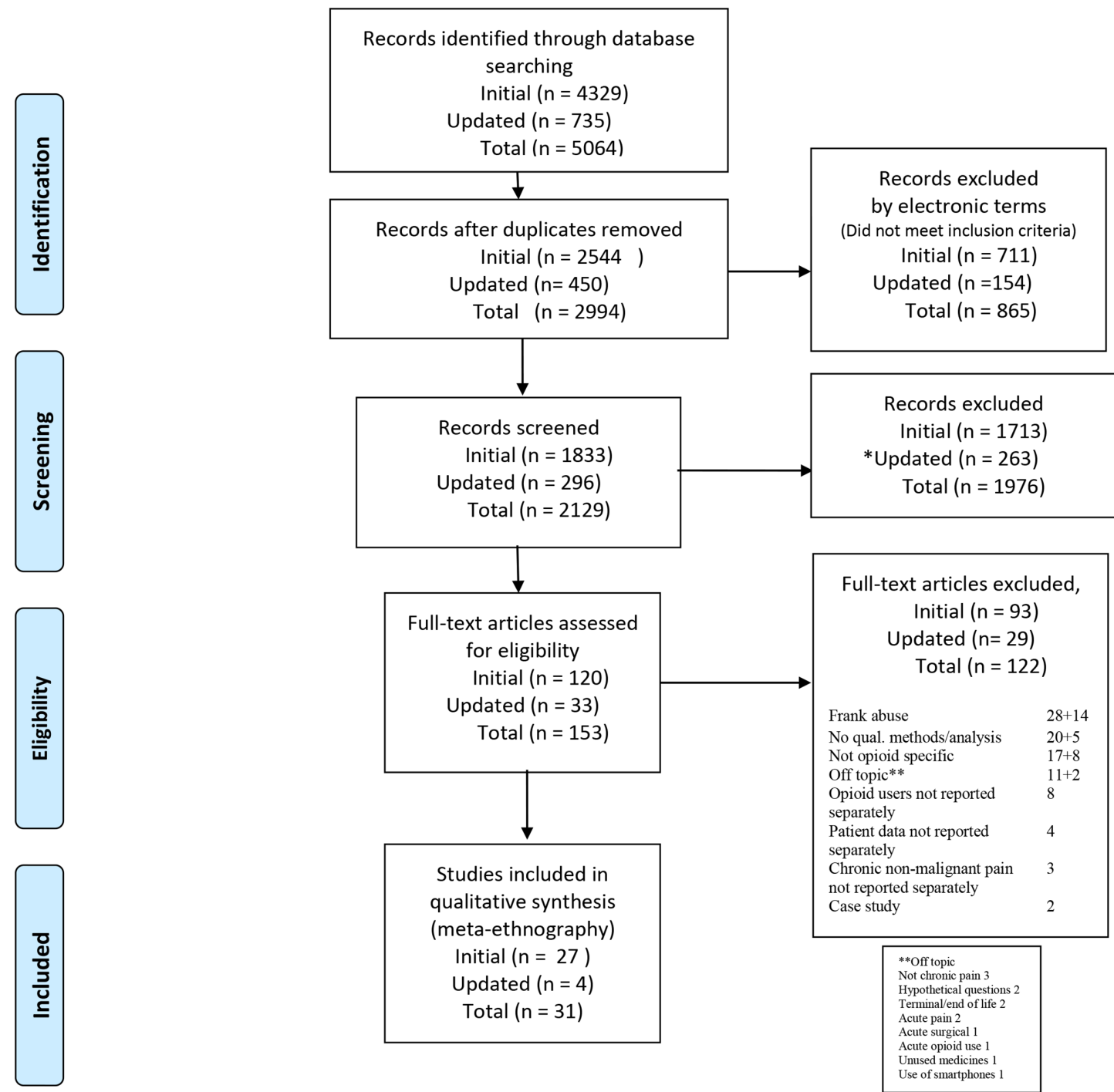

Figure 2 PRISMA flow diagram. ${ }^{52}$ PRISMA, Preferred Reporting Items for Systematic Reviews and Meta-Analyses.

the morphine because I didn't want to be like that. (Zheng et al, p1832) ${ }^{18}$

Some spoke of underusing or were keen to reduce their medications when possible. There was a dislike of being on long-term medication and some thought that it would not relieve their pain.

I don't want to do that [take more morphine]. I want to stay on as little as I possibly can because there might come a time when I need more and I don't want to be on high doses. I've always tried to keep it at a minimum amount of tablets each day.... (Blake et al, p105) ${ }^{17}$

Even though some were reluctant, there were other instances of dramatic improvement in people's lives. This then weighted their choice to stay on the opioids.

I mean it is just like a miracle as far as I am concerned. It is like knowing it [the pain] is there but you have the instruments to prevent it from getting out and [be]coming a roaring demon. (Vallerand and Nowak, p170) ${ }^{19}$

But opiates, that's my way of life. There would be no life if I didn't have this. And I thank God for them because without them I'd be...well I wouldn't be. I just couldn't go on. I would have committed suicide a long time ago. And I say that truthfully cause you could not live like that, with that constant, constant pain. But, with the opiates it's made it possible to be able to have a part of a life, you know. (Brooks et al, p20) $)^{20}$

\section{Understanding opioids: the good and the bad}

This describes patients' knowledge or understanding about opioids which had generally been acquired ad hoc and slowly over time, from pharmacists, patient package inserts in their medication, leaflets, the internet, television programmes and from doctors, especially doctors at the pain clinic. 
Table 2 Confidence in review findings: GRADE-CERQual assessment

\begin{tabular}{|c|c|c|c|c|c|}
\hline Review findings & $\begin{array}{l}\text { Studies contributing } \\
\text { (see table } 1 \text { column } 1 \text { for } \\
\text { study number) }\end{array}$ & $\begin{array}{l}\text { Methodological } \\
\text { limitations (study } \\
\text { number) }\end{array}$ & $\begin{array}{l}\text { Relevance (see } \\
\text { table } 1 \text { end } \\
\text { column) }\end{array}$ & Coherence & $\begin{array}{l}\text { Adequacy of } \\
\text { data }\end{array}$ \\
\hline $\begin{array}{l}\text { Reluctant users with little } \\
\text { choice }\end{array}$ & $\begin{array}{l}1,3,4,5,6,7,17,18,21 \\
22,26,27,30 \text { (13 studies) }\end{array}$ & $\begin{array}{l}11 \text { no concerns. } \\
2 \text { minor concerns }(18 \text {, } \\
22) \text {. }\end{array}$ & $\begin{array}{l}5 \text { Relevant. } \\
6 \text { Partial. } \\
2 \text { Indirect. }\end{array}$ & No concerns. & No concerns. \\
\hline $\begin{array}{l}\text { Understanding opioids: the } \\
\text { good and the bad }\end{array}$ & $\begin{array}{l}1,3,7,9,10,11,15,16,17 \\
23,25,27,29 \text { (13 studies) }\end{array}$ & $\begin{array}{l}12 \text { no concerns. } \\
1 \text { minor concerns (23). }\end{array}$ & $\begin{array}{l}6 \text { Relevant. } \\
6 \text { Partial. } \\
1 \text { Indirect. }\end{array}$ & No concerns. & No concerns. \\
\hline $\begin{array}{l}\text { A therapeutic alliance: not } \\
\text { always on the same page }\end{array}$ & $\begin{array}{l}1,2,3,4,5,7,9,10,11,13 \\
14,15,16,17,18,19,20 \\
21,22,23,24,25,26,28 \\
29,31 \text { (26 studies) }\end{array}$ & $\begin{array}{l}23 \text { no concerns. } \\
3 \text { minor concerns }(18 \text {, } \\
22,23) \text {. }\end{array}$ & $\begin{array}{l}12 \text { Relevant. } \\
11 \text { Partial. } \\
3 \text { Indirect. }\end{array}$ & No concerns. & No concerns. \\
\hline $\begin{array}{l}\text { Stigma: feeling scared } \\
\text { and secretive but needing } \\
\text { support }\end{array}$ & $\begin{array}{l}1,2,3,4,7,9,10,14,16 \\
17,18,20,21,22,23,24 \\
27,28,31 \text { (19 studies) }\end{array}$ & $\begin{array}{l}16 \text { no concerns. } \\
3 \text { minor concerns }(18 \text {, } \\
22,23) \text {. }\end{array}$ & $\begin{array}{l}10 \text { Relevant. } \\
8 \text { Partial. } \\
1 \text { Indirect. }\end{array}$ & No concerns. & No concerns. \\
\hline
\end{tabular}

GRADE-CERQual, Grading of Recommendations Assessment, Development and Evaluation working group - Confidence in Evidence from Reviews of Qualitative research.

When you see it in the media, when you see it on the television, you think if you're taking regular morphine you must be in a pretty bad way, you know. (Blake et al, p103) ${ }^{17}$

I always ask before I go on a medication, what are the side effects, I was told I may experience constipation; nothing else was explained to me. (Paterson $e t$ al, $\mathrm{p} 721)^{21}$

There was often poor knowledge about using opioids for chronic pain, and about addiction, overdose risk and side effects.

There's not too much education about it [overdose]...When I first started taking it [the opioid medication], no one told me about OD [overdose] or anything about that. Because I was taking it not [as] prescribed...I was just like when I felt pain I would just take like five or six of them or whatever. Then at the end, I'd run out. (Mueller et al, p279) ${ }^{22}$

Patients often had to defend their usage and this added to their stress especially when they felt their healthcare professionals lacked an understanding of the place for

\begin{tabular}{ll}
\hline Table 3 GRADE-CERQual component scoring \\
\hline Methodological limitations. & No or very minor concerns. \\
Coherence. & Minor concerns. \\
Adequacy of data. & Moderate concerns. \\
& Serious concerns. \\
Relevance. & Relevant. \\
& Partial. \\
& Indirect. \\
& Uncertain. \\
\hline
\end{tabular}

GRADE-CERQual, Grading of Recommendations Assessment, Development and Evaluation working group - Confidence in Evidence from Reviews of Qualitative research. opioids in the treatment of CNMP or were cautious about using them.

The concern is that if they increase my opioid dosage, I could stop breathing. It's ridiculous. (Frank et al, p1841 $)^{23}$

There are still a lot of doctors out there that are against it. They think it is bad. Bad medicine. Bad practice. (Vallerand and Nowak, p129) ${ }^{24}$

In contrast, some people felt well informed, which either produced more concern or gave the patients confidence in their opioid regimen.

... and from what I've read up, because I like to, sort of, keep on top of things, that it's an opium based drug, so you will build up some tolerance and you will build up [becomes tearful] And you will potentially become sort of addicted to it, if you like. (McCrorie et al, p3 $)^{25}$

Under Dr A [pain clinic] I've learnt more. And my concern has been, well it was initially the possibility of addiction, but she has assured me that I'm not showing no signs of addiction at all. I may have some withdrawal problems. (Paterson et al, p721) ${ }^{21}$

\section{A therapeutic alliance: not always on the same page}

This describes a therapeutic alliance or the relationship between patients and their healthcare providers, which was considered important.

Overall there was a feeling that healthcare professionals and patients were often 'not on the same page' about opioid usage.

My family doctor...does not want me to be dependent on heavy pain meds, so I am intensely miserable $99 \%$ of the time. (Warms et al, p252) ${ }^{26}$ 
Table 4 Themes apparent in each study

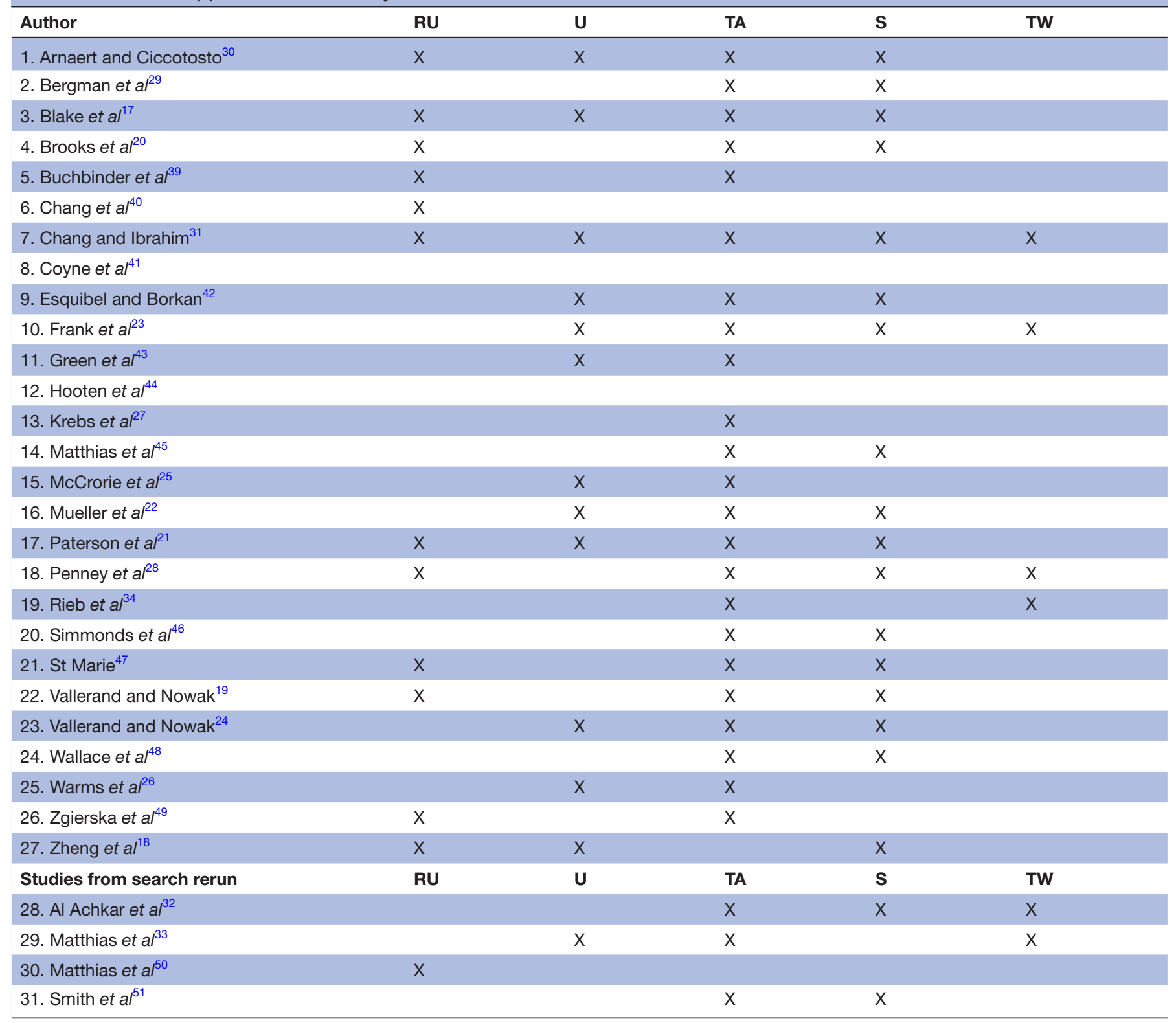

$\mathrm{RU}$, reluctant users with little choice; S, stigma: feeling scared and secretive but needing support; TA, a therapeutic alliance: not always on the same page; TW, the challenges of tapering or withdrawal; $U$, understanding opioids: the good and the bad; $X$, theme present in the paper.

Some patients felt they were not listened to and were frustrated by a lack of empathy from physicians regarding their pain experience.

I frequently have difficulty with the residents (doctors in training) explaining why these drugs, this many drugs...Finally Dr. [family physician] wrote a note in my file - stop harassing [participant's name]. This is what she gets and why she gets it. And they did stop but it was inconvenient. For instance, they would not prescribe me three months at a time. I would be dispensed one month at a time. And for someone who had been taking the same drugs for 10 years I found that condescending. (Brooks et al, p18) ${ }^{20}$
A reluctance to prescribe from general practitioners and pharmacists and the use of opioid contracts or a restriction of medication were often considered punitive.

It kind of made me feel like I was doing something wrong, which I wasn't, but I signed a contract. You know, what would I be without my meds? (Krebs $e t$ al, p1152) $)^{27}$

And I told my doctor that, that I wanted so I could sleep through the night. And now he, well, I'll give you 10 , but it's got to last. Like he treats me like a drug addict. (Penney et $a l$, p6) ${ }^{28}$

The healthcare system often worked against a therapeutic alliance with lack of continuity or care or frequent 
visits, which fed into mistrust. Patients complained that provider turnover affected their ability to receive individualised care; conversations about pain and treatment options often had to be started over again from scratch.

I don't have the same doctor long enough to know. (Bergman et al, p1693) ${ }^{29}$

However having blood or urine tests for levels of opioids and regular checks were seen by some as being cared for.

I would say, 'I have this agreement and you don't have to sign it if you don't want to, but I would like to go over it with you. These are suggestions because this medication is addictive, it is dangerous, and I just want to make sure you're aware.' I think if you really want to make it where people are not hostile, say they have to have a urine test every 6 months, everybody, and that 'it's a policy because we care about all of you.' (Krebs et al, p1152) ${ }^{27}$

Some talked of the need for good relationships built on trust, shared decision making and knowledgeable specialists who communicate well.

I wouldn't say I researched it to that depth, you know, I read a little bit about, and asked a lot of questions at my doctor, and then we decided. (Paterson $e t a l$, p722) $)^{21}$

\section{Stigma: feeling scared and secretive but needing support}

This describes feelings of stigma and fear which people expressed directly in relation to their opioid usage. This includes people's negative attitudes from family, medical professionals and work colleagues which lead to them feeling stigmatised and judged for taking opioids.

So I'm constantly trying to clean up because I think people are going to judge me. 'Oh, because she's on all this medication, ooh, she can't look after her children.' (Paterson et al, p724) ${ }^{21}$

As soon as you mention to someone that you are on pain medication it's, 'Oh my god, you've got to get off it.' It is viewed as weak. Somehow I am weak for being on this medication. (Vallerand and Nowak, p128) ${ }^{24}$

To protect themselves, some chose to keep their opioids a secret.

But you know, after 2 years of pain, you are physically exhausted, mentally exhausted and depressed. So, I take my medication and I hide it at the bottom of my drawer. It's my secret life. It's always a secret, and I've got to hide it and not tell anyone. (Vallerand and Nowak, p169) ${ }^{19}$

Some people made a conscious decision about who they could tell and who they could not due to negative reactions. Relationships suffered when patients felt unsupported.
My son told me I was a drug addict. He did. He really did. He was to the point, he didn't know what he could do for me. It really was that bad. (Vallerand and Nowak, p128) ${ }^{24}$

I had originally told my sister and she was very concerned. Then she said, 'As long as you don't stay on them.' She thought it was OK if I did it for a while but as long as I didn't stay on them. So I just sort of never told her. And she never asked. (Vallerand and Nowak, p128) ${ }^{24}$

Although some seemed confident in using opioids, mostly people spoke about fears such as addiction and uncontrolled pain. Feeling supported validated their choices and experiences and lessened some of their fears and concerns.

And at the end, my partner says-we sat down there and he goes 'Stay on them.' ...I've always spoke to my partner, and if he's been unsure-we've both been unsure, we've both gone into the doctor together to ask questions. (Zheng et al, p1834) ${ }^{18}$

my wife wanted me to take this medication. She was like: let's go for it. (Arnaert and Ciccotosto, p26) ${ }^{30}$

\section{The challenge of tapering/withdrawal from opioids}

Four papers ${ }^{23}$ 31-33 explored patients' experiences of tapering or withdrawing as their main content. Two further papers ${ }^{2834}$ addressed it as a more peripheral issue (see CERQual ratings in table 2). This describes the challenges and profound effects of tapering or withdrawing from opioids.

Tapering and withdrawing from opioids could be challenging and provoke anxiety.

I have a tremendous fear in a doctor saying I want you to taper off the methadone and get totally off the methadone with no alternative whatsoever. I think that would be an irrational decision by a doctor, and I probably wouldn't take that advice. (Frank et al, p1842) $)^{23}$

This anxiety could be alleviated by support from a trusted healthcare provider or other person.

The best thing about it was that nobody acted like I was a bad person because I was on these medications and was having to be going through this really slow process of coming down off of them. (Frank et $a l, \mathrm{p} 1843)^{23}$

Successful tapering was described as a collaborative agreement between the healthcare professional and the patient.

She put me down to 2 and a half [pills per day]. Then she said, okay, we'll go down to half a pill. I told her I didn't think that just 2 a day would do it, and she said okay, we'll try 2 and a half, are you agreeable with that? I said that's fine. I mean, we can discuss stuff. It doesn't have to be a disagreement because we can 
talk about it. It's not an argument. We're 2 adults having a conversation, figuring out what to do. (Matthias et al, $\mathrm{p} 1368)^{33}$

However, not all people experienced joint decision making when tapering.

I just don't feel that he's understanding. he don't seem to care what I'm saying, because he's lowering it down anyway, even though I've told him...that I didn't agree with it being lowered. (Matthias et al, p1369) ${ }^{33}$

For those in the USA, prescribing policies, advising clinicians to monitor and decrease opioid use, and the legislation to enforce these policies made those taking opioids feel as if they were 'a public health problem'. This could have a negative effect on the doctor-patient relationship and leave the patient feeling disempowered. This was compounded when opioids had been withdrawn by legislation. ${ }^{31} 32$

I have to struggle, suffer, to make the next the next time that I can get my medicine. And I don't think that's fair to me because if I can take my medicine a little more regularly, I would be able to do more...I don't think that the law, people, politicians, or anybody should be able to tell anybody that's in pain what type of medicine they can take. (Al Achkar et $a l, \mathrm{p} 7)^{32}$

That kinda got me mad, cause I thought well you know...they're taking it off the market because of people abusing it...It's not fair to us, you know...I think the government was wrong to...pull them off the market, you know, because of people abusing them, no like they weren't looking at the people that need them...But I think it's really unfair that people that really do need them can't get them. (Chang and Ibrahim, 2017, p3) ${ }^{31}$

\section{Overarching theme: constantly balancing}

After considering the fives themes, an overarching theme emerged: 'Constant balancing'. The theme reluctant users with little choice describes the need to balance the pros and cons of starting opioids and the need to balance having pain with their hesitancy to use opioids.

I don't really like being on a lot of tablets, I've never been a tablet person, um...but I mean I can't have the pain either so it's one evil outdoing the other evil. (Paterson et al, p723) ${ }^{21}$

Studies describe balancing the dose for pain management with their side effects to allow them to function. Participants constantly weighed up the effects on their life: dealing with an internal conflict of unresolved pain versus necessary medication, being opioid free versus having uncontrolled pain, and balancing other stressors against opioid dose changes.
If you're going to be able to walk, and you take one pain pill so you can walk and live life, you're going to do it, even though you may not like it. (Penney et al, p6) $)^{28}$

The theme stigma: feeling scared and secretive but needing support describes the need to balance their hopes for relief with fear of side effects, and also to balance whether or not to disclose their opioid use with the risk of being labelled a 'drug seeker' versus having unrelieved pain.

I do it for my own protection by not telling them because I see how they react by reading something in the paper...and it's just their ignorance. And I don't have time. Well they know what's going on but they don't get it to this day. So you have to pick your battles.... (Brooks et al, p19) ${ }^{20}$

The theme understanding opioids: the good and the bad showed people had different levels of understanding, but weighed up their decisions and trade-offs against their pain relief.

It's, it's got a good and bad side, morphine.... When I take it, it works really, really well but it makes you feel rather sick, umm, rather spaced out and thinking wise, umm, it outcomes more on the other, do I want to be sick or do I want to cry with pain? So I'd rather be sick but it is a very, very good painkiller. (Blake $e t$ $a l, \mathrm{p} 105)^{17}$

The therapeutic alliance theme showed that often it was evident that they were 'not on the same page', with them balancing the advice from their doctors with what they wanted.

[My provider] said you could die any time, and my husband and I said, well, we realize that, but because of the pain, you know, we were willing to take that risk that I would die from the narcotic medication. (Frank et al, p1841) ${ }^{23}$

It also meant that there were multiple barriers to the process of decreasing opioids due to this constant balancing act, which is described in the theme the challenge of tapering/withdrawal from opioids.

I will tell her, if I do come off this medication, there are going to be consequences. I can't walk as often, I can't stand as long, I just can't do it.... (Vallerand and Nowak, p169) ${ }^{19}$

\section{DISCUSSION}

Our five themes were (1) reluctant users with little choice; (2) understanding opioids: the good and the bad; (3) a therapeutic alliance: not always on the same page; (4) stigma: feeling scared and secretive but needing support; and (5) the challenge of tapering or withdrawal. An overarching theme of 'constantly balancing' emerged from the data. These themes all had positive and negative aspects, although the negative were more prevalent . 
We present a line of argument of how complex it is for the patient to balance decisions at every stage of their journey. First is their reluctance to start taking opioids but feeling they had no option. Patients are given opioids for CNMP often as a last resort when all other treatment has failed and their lives are so profoundly affected that they talk of a desperation, that they would literally 'try anything'. Patients spoke about not being given any detailed information about opioids and that they had learnt more about them over time from different sources. This varied understanding about opioids and their side effects can affect the decisions that people make. Patients reported the need to keep the dosage of opioids as low as possible and often that they were not at risk of addiction or overdose if they were taking them as prescribed. Even those who felt they may be addicted sometimes viewed this as an acceptable trade-off for pain relief. Our findings indicate that patient desperation combined with inadequate information from healthcare professionals could trigger the prescription of opioids. It may be that delivering accurate information about the potential side effects and limited efficacy of opioids for chronic pain management would reduce the use of opioids.

Our findings demonstrate that the stigma surrounding how patients feel about being on opioids can be compounded by the judgements of others. Although patients often describe themselves in terms of 'reluctant users', if they experienced the benefits of opioids through decreased pain and thus increased function they are often too scared to reduce opioids and return to a life of potentially unmanaged pain.

Our findings suggest that clinicians and patients with chronic pain are not always 'on the same page'. The theme a therapeutic alliance captures the positives, but also the tensions and mismatches of perceptions held by healthcare providers who are attempting to limit dose escalation, and patients who may view constant dose escalation as an acceptable trade-off for reducing relentless pain. The therapeutic alliance is a robust theme supported by 26 of the 31 studies included. This is not surprising as patients rely on their healthcare professionals to prescribe opioids. This finding resonates with qualitative evidence syntheses exploring the experience of patients ${ }^{13}$ and healthcare professionals. ${ }^{35}$ It seems clear that joint decision making is important for appropriate healthcare; however, our findings suggest that there are instances of mistrust on both sides. A qualitative evidence synthesis exploring clinicians' experience of prescribing opioids for chronic pain demonstrates that the process of prescribing opioids is not straightforward for clinicians who face a complex decision: "Should I shouldn't I' prescribe opioids for CNMP?"35 They also demonstrate that clinicians must walk a fine line to balance the pros and cons of opioids while also maintaining patient trust. This suggests that both patients and healthcare professionals find dealing with prescribing/taking opioids for CNMP is complex and involves balancing and trade-offs.
Current guidance from the Royal College of Anaesthetists in the UK and the Centers for Disease Control and Prevention in the USA advocates a preference for non-opioid therapies in the treatment of CNMP. ${ }^{36}$ If a clinician feels that opioids are indicated, then they recommend a low dose for a short duration, which should be assessed for effectiveness and regularly evaluated for benefits and harms. All but four studies in this review are between 2005 and 2017, prior to these guidelines. Opioid contracts in some areas of the USA and Canada can make patients feel stigmatised and judged, and this effect can be moderated by a good therapeutic relationship, and reframing these as agreements rather than contracts. ${ }^{37}$ Some physicians may view contracts/ agreements as necessary to guard against uncontrolled dose escalation, repeated demands for replacement of lost or misplaced medication, subversion and illicit opioid intake. This finding resonates with Toye $e t a l^{35}$, who described the moral boundary work and social guardianship that clinicians associate with opioid prescription. Our findings suggest that this role may not contribute to an effective therapeutic partnership.

\section{Limitations of this study}

A majority of the studies are from the USA, and the findings need to be taken in the context of its health and social care systems. Most of the articles in this qualitative synthesis were published or the research was conducted before the impact of the opioid epidemic became clear to regulators and the medical profession. Some papers discussed using opioids as a last resort, although the opioid epidemic, especially in the USA, indicates that the threshold for prescribing opioids was low until recent initiatives to discourage prescribing long-term opioids for chronic pain. ${ }^{38}$ Not all studies gave morphine equivalent data, so we cannot determine what proportion were taking high, medium or low doses. We acknowledge that our interpretation of the data might have been influenced by the current, much more critical perception of opioid use for CNMP. Further evidence is needed to find out if these themes are universal for developed countries or whether there are important differences.

Our conceptual framework highlights that patients need to constantly balance and to consider the pros and cons of taking opioids. This can have a profound effect on people's relationships with their family, friends and healthcare providers and their perceived standing in the community, which is reflected in their careful balancing of disclosure. The therapeutic alliance and having a clear understanding of all the positive and negative aspects of opioids were important factors that underpinned their ability to maintain this fragile balance. This balance might also affect a person's desire or ability to taper or withdraw from opioids.

The GRADE-CERQual ratings (table 2) revealed we had confidence in the findings, with only a few minor concerns and no moderate or serious concerns. 


\section{CONCLUSIONS AND RECOMMENDATIONS FOR FUTURE RESEARCH}

The first meta-ethnography on this topic revealed a constant balancing and a life in flux in an effort to maintain participation in life and relationships. These are important features of opioid use for CNMP. To maintain this delicate balance, they often need support from family or clinicians; however, this balance can be upset by the feeling of being judged by this same potential support system or peers and society at large through the media. The therapeutic alliance with healthcare professionals, the extent of people's understanding, as well as the stigma attached to opioid use need to be navigated by people who are often reluctant to be on opioids in the first place.

\section{Twitter Harbinder Kaur Sandhu @DrHSandhu}

Acknowledgements We would like to thank Samantha Johnson, an academic librarian who helped with the electronic searches, and Dr Stephanie Tierney, who helped to screen the citations.

Contributors VPN and KS contributed to the review concept and design as part of the I-WOTCH process evaluation team. HKS, SE, MU and KS were involved in the design of the I-WOTCH study. VPN, KS and FT screened the search results or extracted the data, and conducted the analysis and synthesis. All authors contributed to data interpretation, revised the final manuscript critically for important intellectual content and appraised the final manuscript. VPN prepared the final manuscript and is the corresponding author.

Funding This project was funded by the National Institute for Health Research, Health Technology Assessment (project number 14/224/04). The views and opinions expressed therein are those of the authors and do not necessarily reflect those of the HTA, NIHR, NHS or the Department of Health.

Competing interests KS has undertaken other meta-ethnographies and was on the NIHR HS\&DR Board until January 2018. SE is an investigator on a number of NIHR and industry-sponsored studies. He received travel expenses for speaking at conferences from professional organisations. SE consults for Medtronic, Abbott, Boston Scientific and Mainstay Medical, none in relation to opioids. SE is Chair of the BPS Science and Research Committee. SE is Deputy Chair of the NIHR CRN Anaesthesia Pain and Perioperative Medicine National Specialty Group. SE's department has received fellowship funding from Medtronic, as well as nurse funding from Abbott. HKS is Director of Health Psychology Services, providing psychological services for a range of health-related conditions. MU was Chair of the NICE accreditation advisory committee until March 2017, for which he received a fee. He is chief investigator or coinvestigator on multiple previous and current research grants from the UK National Institute for Health Research and Arthritis Research UK, and is a coinvestigator on grants funded by the Australian NHMRC. He is an NIHR senior investigator. He has received travel expenses for speaking at conferences from professional organisations hosting conferences. He is a director and shareholder of Clinvivo, which provides electronic data collection for health services research. He is part of an academic partnership with Serco related to return to work initiatives. He is a coinvestigator on a study receiving support in kind from Stryker. He has accepted honoraria for teaching from CARTA. He is an editor of the NIHR journal series, and a member of the NIHR Journal Editors Group, for which he receives a fee.

Patient consent for publication Not required.

Ethics approval Not applicable - a review

Provenance and peer review Not commissioned; externally peer reviewed.

Data availability statement Data are available on reasonable request.

Open access This is an open access article distributed in accordance with the Creative Commons Attribution 4.0 Unported (CC BY 4.0) license, which permits others to copy, redistribute, remix, transform and build upon this work for any purpose, provided the original work is properly cited, a link to the licence is given, and indication of whether changes were made. See: https://creativecommons.org/ licenses/by/4.0/.

\section{ORCID iD}

Vivien P Nichols http://orcid.org/0000-0002-3372-1395
REFERENCES

1 Breivik H, Collett B, Ventafridda V, et al. Survey of chronic pain in Europe: prevalence, impact on daily life, and treatment. Eur J Pain 2006;10:287-333.

2 Nahin RL. Estimates of pain prevalence and severity in adults: United States, 2012. The Journal of Pain 2015;16:769-80.

3 Els C, Jackson TD, Kunyk D, et al. Adverse events associated with medium- and long-term use of opioids for chronic non-cancer pain: an overview of Cochrane reviews. Cochrane Database of Systematic Reviews 2017.

4 Gomes T, Tadrous M, Mamdani MM, et al. The burden of opioidrelated mortality in the United States. JAMA Network Open 2018;1:e180217.

5 Furlan ADet al. Opioids for chronic noncancer pain: a metaanalysis of effectiveness and side effects. Can Med Assoc J 2006;174:1589-94.

6 Busse JW, Wang L, Kamaleldin M, et al. Opioids for chronic noncancer pain: a systematic review and meta-analysis. JAMA 2018;320:2448-60.

7 Sandhu HK, Abraham C, Alleyne S, et al. Testing a support programme for opioid reduction for people with chronic nonmalignant pain: the I-WOTCH randomised controlled trial protocol. BMJ Open 2019;9:e028937.

8 Nichols VP, Abraham C, Eldabe S, et al. Process evaluation protocol for the I-WOTCH study: an opioid tapering support programme for people with chronic non-malignant pain. BMJ Open 2019;9:e028998.

9 Noblit GW, Hare RD. Meta-ethnography: synthesizing qualitative studies. Sage 1988.

10 France EF, Cunningham M, Ring N, et al. Improving reporting of meta-ethnography: The eMERG e reporting guidance. $J$ Adv Nurs 2019;75:1126-39.

11 Manchikanti L, Helm S, Fellows B, et al. Opioid epidemic in the United States. Pain Physician 2012;15:ES9-38.

12 CASP. Critical appraisal skills programme. Public Health Resource Unit: Oxford, 2006.

13 Toye F, Seers K, Barker KL. Meta-ethnography to understand healthcare professionals' experience of treating adults with chronic non-malignant pain. BMJ Open 2017;7:e018411.

14 Lewin S, Booth A, Glenton C, et al. Applying GRADE-CERQual to qualitative evidence synthesis findings: introduction to the series. Implementation Sci 2018;13:2.

15 Lewin S, Bohren M, Rashidian A, et al. Applying GRADE-CERQual to qualitative evidence synthesis findings-paper 2: how to make an overall CERQual assessment of confidence and create a summary of qualitative findings table. Implementation Sci 2018;13:10.

16 Schutz A. Collected papers. The Hague: Martinus Nijhoff, 1962: Vol. 1. 2.

17 Blake S, Ruel B, Seamark C, et al. Experiences of patients requiring strong opioid drugs for chronic non-cancer pain: a patient-initiated study. Br J Gen Pract 2007;57:101-8.

18 Zheng Z, Paterson C, Ledgerwood K, et al. Chaos to hope: a narrative of healing. Pain Medicine 2013;14:1826-38.

19 Vallerand A, Nowak L. Chronic opioid therapy for nonmalignant pain: the patient's perspective. Part I-Life before and after opioid therapy. Pain Management Nursing 2009;10:165-72.

20 Brooks EA, Unruh A, Lynch ME. Exploring the lived experience of adults using prescription opioids to manage chronic noncancer pain. Pain Research and Management 2015;20:15-22.

21 Paterson C, Ledgerwood K, Arnold C, et al. Resisting prescribed opioids: a qualitative study of decision making in patients taking opioids for chronic noncancer pain. Pain Medicine 2016;17:717-27.

22 Mueller SR, Koester S, Glanz JM, et al. Attitudes toward naloxone prescribing in clinical settings: a qualitative study of patients prescribed high dose opioids for chronic non-cancer pain. J Gen Intern Med 2017;32:277-83.

23 Frank JW, Levy C, Matlock DD, et al. Patients' Perspectives on Tapering of Chronic Opioid Therapy: A Qualitative Study. Pain Medicine 2016;17:1838-47.

24 Vallerand A, Nowak L. Chronic opioid therapy for nonmalignant pain: the patient's perspective. Part II--Barriers to chronic opioid therapy. Pain Manag Nurs 2010;11:126-31.

25 McCrorie C, Closs SJ, House A, et al. Understanding long-term opioid prescribing for non-cancer pain in primary care: a qualitative study. BMC Fam Pract 2015;16:1-9.

26 Warms CA, Marshall HM, Hoffman AJ, et al. There are a few things you did not ask about my pain: writing on the margins of a survey questionnaire. Rehabilitation Nursing 2005;30:248-56.

27 Krebs EE, Bergman AA, Coffing JM, et al. Barriers to guidelineconcordant opioid management in primary care--a qualitative study. J Pain 2014;15:1148-55. 
28 Penney LS, Ritenbaugh C, DeBar LL, et al. Provider and patient perspectives on opioids and alternative treatments for managing chronic pain: a qualitative study. BMC Family Practice 2017;17:1-15

29 Bergman AA, Matthias MS, Coffing JM, et al. Contrasting tensions between patients and PCPs in chronic pain management: a qualitative study: table 1. Pain Medicine 2013;14:1689-97.

30 Arnaert A, Ciccotosto G. Response phases in methadone treatment for chronic nonmalignant pain. Pain Manag Nurs 2006;7:23-30.

31 Chang F, Ibrahim S. Perceptions of community-dwelling patients and their physicians on OxyContin $®$ discontinuation and the impact on chronic pain management. Pain Research and Management 2017;2017:1-11.

32 Al Achkar M, Revere D, Dennis B, et al. Exploring perceptions and experiences of patients who have chronic pain as state prescription opioid policies change: a qualitative study in Indiana. BMJ Open 2017;7:e015083.

33 Matthias MS, Johnson NL, Shields CG, et al. "I'm Not Gonna Pull the Rug out From Under You": Patient-Provider Communication About Opioid Tapering. J Pain 2017;18:1365-73.

34 Rieb LM, Norman WV, Martin RE, et al. Withdrawal-associated injury site pain (WISP): a descriptive case series of an opioid cessation phenomenon. Pain 2016;157:2865-74.

35 Toye F, Seers K, Tierney S, et al. A qualitative evidence synthesis to explore healthcare professionals' experience of prescribing opioids to adults with chronic non-malignant pain. BMC Fam Pract 2017;18:94

36 CDC. Cdc guideline for prescribing opioids for chronic pain. accessed 12 Apr 20192019.

37 Tobin DG, Keough Forte K, Johnson McGee S. Breaking the pain contract: a better controlled-substance agreement for patients on chronic opioid therapy. Cleve Clin J Med 2016;83:827-35.

38 Schieber LZ, Guy GP, Seth P, et al. Trends and patterns of geographic variation in opioid prescribing practices by state, United States, 2006-2017. JAMA Netw Open 2019;2:e190665.

39 Buchbinder M, Wilbur R, McLean S, et al. "Is there any way I can get something for my pain?" Patient strategies for requesting analgesics. Patient Educ Couns 2015;98:137-43.

40 Chang Y-P, Wray LO, Sessanna L, et al. Use of prescription opioid medication among community-dwelling older adults with noncancer chronic pain. J Addict Nurs 2011;22:19-24.
41 Coyne KS, Currie BM, Holmes WC, et al. Assessment of a stool symptom Screener and understanding the opioid-induced constipation symptom experience. Patient 2015;8:317-27.

42 Esquibel AY, Borkan J. Doctors and patients in pain: conflict and collaboration in opioid prescription in primary care. Pain 2014;155:2575-82.

43 Green TC, Case P, Fiske H, et al. Perpetuating stigma or reducing risk? perspectives from naloxone consumers and pharmacists on pharmacy-based naloxone in 2 states. J Am Pharm Assoc 2017:57:S19-27.

44 Hooten WM, Vickers KS, Shi Y, et al. Smoking cessation and chronic pain: patient and pain medicine physician attitudes. Pain Practice 2011;11:552-63.

45 Matthias MS, Krebs EE, Bergman AA, et al. Communicating about opioids for chronic pain: a qualitative study of patient attributions and the influence of the patient-physician relationship. Eur J Pain 2014;18:835-43.

46 Simmonds MJ, Finley EP, Vale S, et al. A qualitative study of Veterans on long-term opioid analgesics: barriers and facilitators to multimodality pain management. Pain Medicine 2015;16:726-32.

47 St. Marie B. Primary care experiences of people who live with chronic pain and receive opioids to manage pain. J Am Assoc Nurse Pract 2016;28:429-35.

48 Wallace L, Wexler R, McDougle L, et al. Voices that may not otherwise be heard: a qualitative exploration into the perspectives of primary care patients living with chronic pain. J Pain Res 2014;7:291-9.

49 Zgierska AE, Burzinski CA, Cox J, et al. Mindfulness MeditationBased intervention is feasible, acceptable, and safe for chronic low back pain requiring long-term daily opioid therapy. J Altern Complement Med 2016;22:610-20.

50 Matthias MS, Donaldson MT, Jensen AC, et al. "I Was a Little Surprised": Qualitative Insights From Patients Enrolled in a 12-Month Trial Comparing Opioids With Nonopioid Medications for Chronic Musculoskeletal Pain. J Pain 2018;19:1082-90.

51 Smith CL, Severtsen B, Vandermause R, et al. Seeking Chronic Pain Relief: A Hermeneutic Exploration. Pain Management Nursing 2018;19:652-62.

52 Moher D, Liberati A, Tetzlaff J, et al. Preferred reporting items for systematic reviews and meta-analyses: the PRISMA statement. PLoS Med 2009;6:e1000097. 